\title{
Multilayer RF PCB for Space Applications : technological and interconnections trade-off
}

\author{
M. Paillard ${ }^{1}$, F. Bodereau ${ }^{1}$, C. Drevon ${ }^{1}$, P. Monfraix ${ }^{1}$, J.L. Cazaux ${ }^{1}$ \\ L.Bodin ${ }^{2}$, P. Guyon ${ }^{2}$ \\ ${ }^{1}$ Alcatel Space, 26 Avenue Champollion, BP1187, F-31037 Toulouse, France \\ mathieu.paillard@space.alcatel.fr, Tel : +33 (0)53435 37 73, Fax: +33 (0)5 34356947 \\ ${ }^{2}$ Cimulec, Z.I "les Jonquières", F-57365 Ennery, France \\ lbodin@,cimulec.com, Tel : +33(0)387738673, Fax : +33 (0)387738713
}

\begin{abstract}
Multilayer RF Printed Circuits Boards with embedded passives are complex structures to manufacture and to package while keeping good RF performances. In this paper, technological solutions aimed at simplifying these issues are proposed : the use of thermoset materials instead of thermoplastic laminates and an original interconnection technique based on "RF openings" machined in the edges of the boards. Two breadboards based on these solutions have been developed and demonstrate very $\operatorname{good} R F$ performances between 5 and $15 \mathrm{GHz}$.
\end{abstract}

\section{INTRODUCTION}

The increasing complexity of telecommunication payloads in new $\mathrm{Ku}$ and $\mathrm{Ka}$-band satellites often required multilayer RF Printed Circuit Boards (PCB) for applications such as Beam Forming Network of active antennas or Local Oscillator frequency distribution in RF assemblies [1].

From a technological point of view, the main specifications of these multilayer structures are the following :

- operating frequency in the $[5-15 \mathrm{GHz}]$ range (could be wideband)

- $\quad$ large dimensions (up to 265 x $165 \mathrm{~mm}$ )

- $\quad$ up to 12 layers

- $\quad$ include embedded resistors

- sequential construction...

Beyond these technological aspects, such circuits also have to fulfil high reliability requirements imposed by space harsh environment.

First complex multilayer RF PCB developed at Alcatel Space more than five years ago such as the one illustrated in Fig.1 were manufactured with thermoplastic (i.e. Teflon ${ }^{\circledR}$ (PTFE) based) substrates and prepreg [2]. If these materials usually offer very good RF properties they also have to be handled with care in production because they exhibit low thermo-mechanical stability, require specific treatments for drilling, high lamination temperature... Together with the fragility of embedded resistors, these limitations make manufacturing of PTFEbased multilayer structures difficult. And these difficulties, source of inaccuracies in the final assembly, could in the end turn into degradations of circuits RF performances.

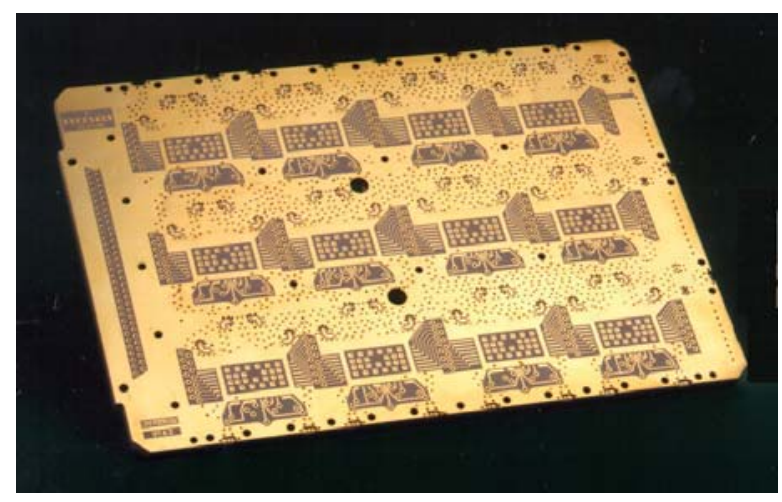

Fig. 1. External view of a 12 layers RF PCB.

Another critical issue with such large and complex circuits deals with their packaging and their integration into space-borne equipments. In particular, poor interconnections of multilayer circuits to their environment could result in non acceptable RF performances at the equipment level.

In order to try to overcome these limitations a new generation of multilayer circuits based on thermoset materials has been developed and characterised. In parallel, improvements of interconnection issues have also been successfully addressed. This results in multilayer RF PCB easier to manufacture but also to integrate in their environment with RF performances comparable to the ones obtained with PTFE-based materials.

\section{THERMOSET BASED RF MULTILAYER PCB}

Thermoset substrates and prepreg for RF purposes are now proposed by several laminates distributors. Such products, initially developed as low cost alternative to PTFE laminates, are based on an hydrocarbon resin system with ceramics fillers and woven glass structure. Their nature allow them to be handled in production with processes close to the ones used for traditional FR4 boards. From an electrical point of view, references available for multilayer applications typically exhibit the following dielectric properties at $10 \mathrm{GHz}$ :

- dielectric constant : $3<\varepsilon_{\mathrm{r}}<3.5$

- dissipation factor : $0.0025<\tan \delta<0.005$ 


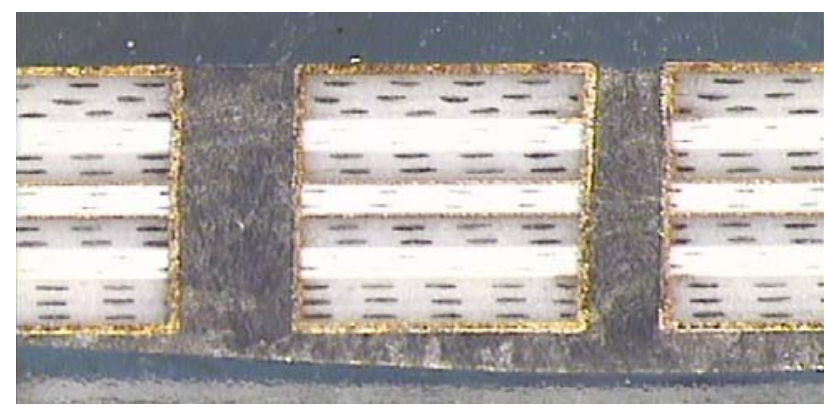

Fig. 2. Cross-section of a 6 layers thermoset-based RF PCB showing the different layers of substrate and prepreg and their woven glass structure.

Even if some PTFE-based substrates could offer lower losses (down to 0.0008), their associated prepregs are comparable to the thermoset ones from this point of view. Hence final assemblies have rather similar dielectric properties with both kind of materials.

The main advantage of thermoset materials over their thermoplastic counterparts deals with their much better in-plane mechanical stability as all substrates and prepregs have woven glass structure (see Fig. 2). They also offer limited $\mathrm{z}$-axis coefficient of thermal expansion (typically a few tenth of $\mathrm{ppm} /{ }^{\circ} \mathrm{C}$ ) compatible with Plated Through Holes (PTH) reliability in multilayer boards.

Last but not least, thermoset prepregs exhibit lower melting temperature $\left(<200^{\circ} \mathrm{C}\right)$ than thermoplastic bonding films $\left(200\right.$ to $\left.300^{\circ} \mathrm{C}\right)$ and by nature do not melt again during sequential laminations. This lower bonding temperature is also a positive aspect for the reliability and the stability of embedded resistances.

All these properties make multilayer structures based on thermoset materials easier to manufacture than PTFEbased ones. Benefits expected in term of better accuracy on final assemblies should translate into better electrical performances of circuits.

\section{RF INTERCONNECTIONS IN MULTILAYER PCB}

RF multilayer PCB designed at Alcatel Space are based on stripline architecture. Then, beyond design and manufacturing challenges, a last issue deals with interconnections of final circuits to their environment while keeping optimised RF performances.

A common solution consists in bringing the RF signal from the inner layers to the top surface of the circuit with PTH. Then coplanar lines are used to route the signal on the board surface. For the last level of interconnection (between the first embedded RF line and the top surface), laser micro-vias is a alternative to PTH we have also successfully used.

These two techniques are relevant to connect RF lines to components or MCM on top of the circuit. However, they could be source of strong limitations for interconnections to the "outside world" (other circuits, connectors...). First, because of inaccuracies in the location of PTH, both between them and with the pads they are supposed to connect in inner layers. But also because of ground plane continuity issues, especially for high layer count structures operating above $10 \mathrm{GHz}$.

To solve these issues, an original alternative consists in manufacturing so-called "RF openings" in the edge of the circuit in order to have a direct access to the RF line in the lower signal level of the circuit (see Fig. 3). These openings are mechanically and laser machined with tight tolerances. To that purpose a new and special process has been developed at the PCB shop focusing on mechanical tolerances and access cleanliness with perfect surface finishing to meet gold wire bonding specifications. This new industrial process can be applied to different substrates and openings surface finishing such as galvanic nickel gold, electroless nickel gold or galvanic tin lead.

Then, interconnection to a connector or another circuit is easily made with, for example, a gold ribbon and the ground plane continuity is guarantee by the bottom face of the circuit in contact with the package structure. This technique also bring a much better reproducibility from a connection to another than PTH-based alternatives, which is of particular interest for RF applications as it could lead to strong tuning time reduction.

\section{MULTILAYER BOARDS EVALUATION}

Two breadboards have been designed, manufactured and tested in order to evaluate benefits of both thermoset materials and "RF openings" : a 3 layers PTFE-based circuit and a 6 layer thermoset-based one. Both circuits include embedded resistors, RF openings and common RF test structures such as $50 \Omega$ lines, Wilkinson power dividers, $-3 \mathrm{~dB}$ attenuators...

RF test structures layouts have been optimised with Agilent Serie IV and 3D electromagnetic simulations were performed with HFSS to design the build-up architecture and optimise interconnections at the RF openings level.

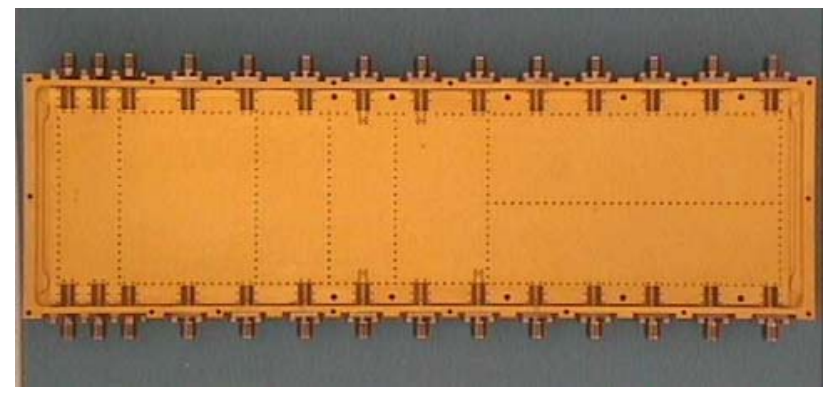

Fig. 4. : 6 layers thermoset-based board with its package. 

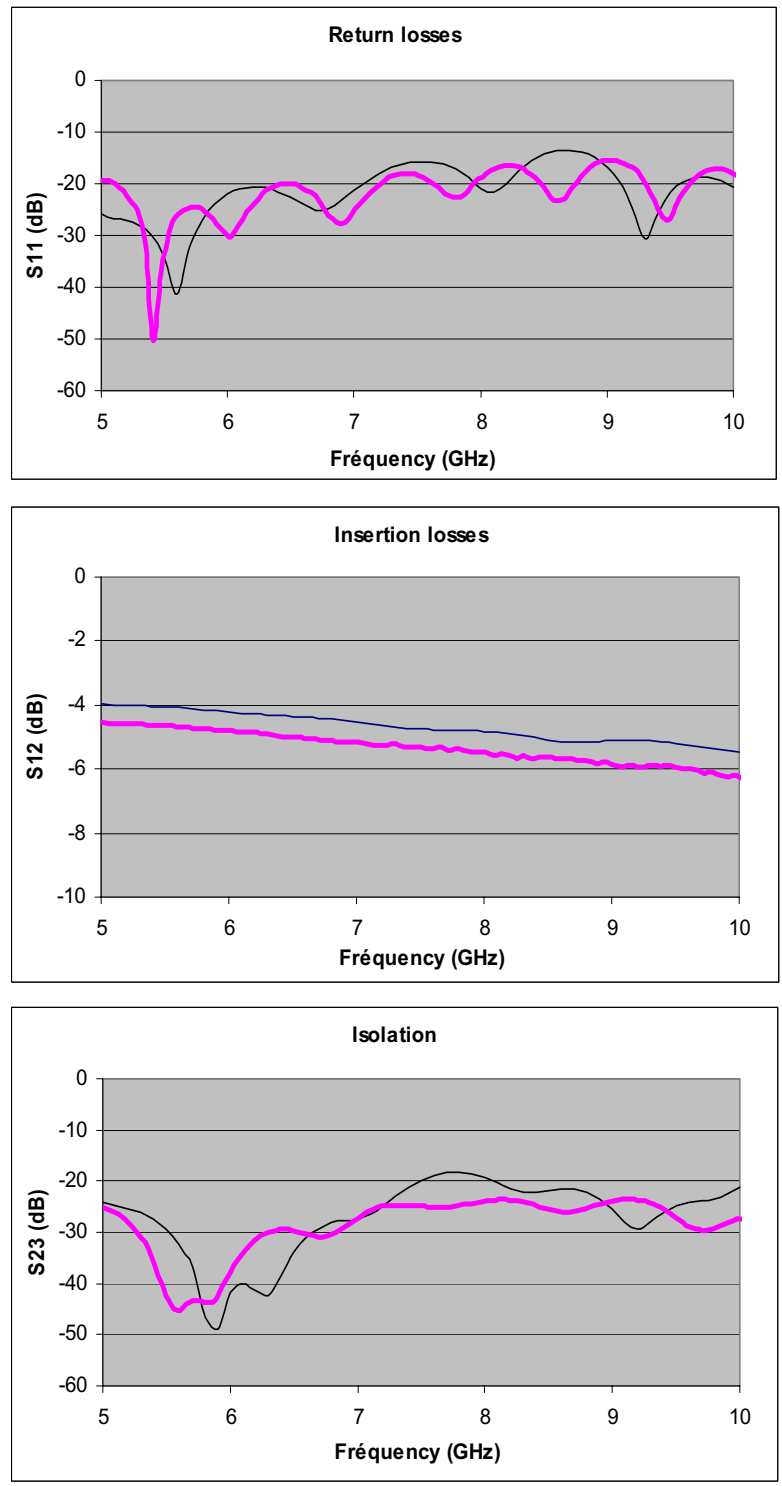

Fig. 5. S-parameters simulation (thin line) and measurements (thick line) of the 6 layers thermoset-based RF board.

For RF measurements purposes, multilayer circuits were integrated in an aluminium package with $\mathrm{K}$ connectors as illustrated in Fig.4. Gold ribbon were used to interconnect RF lines at the "RF openings" level to the connectors sliding contacts. S-parameters measurements were performed between 5 and $15 \mathrm{GHz}$ with a network analyser.

Fig. 5 shows the comparison between S-parameters simulations and measurements of a Wilkinson power divider from the 6 layers thermoset board optimised for the $[5-10 \mathrm{Ghz}]$ frequency band. The good agreement between measurements and simulations demonstrates the very good predictability of our models, including RF performances of the transition at the "RF openings" level.

From a material comparison point of view, Fig. 6 exhibits S-parameters measurements of $50 \Omega$ lines from the two different circuits on the $5-15 \mathrm{GHz}$ frequency band. Both structures exhibit good and rather similar performances. One can note that return losses keep below
$-20 \mathrm{~dB}$ on the whole frequency band : on a test structure as simple as a $50 \Omega$ line, this clearly demonstrates the good impedance matching and ground plane continuity at the interconnection level between the multilayer board and its package, i.e. the benefit and the wideband character of the "RF opening" solution.

Insertion losses range from $-1 \mathrm{~dB}$ at $5 \mathrm{GHz}$ to $-2.2 \mathrm{~dB}$ at $15 \mathrm{GHz}$ for both multilayer circuits in spite of the slightly higher dielectric losses from the thermoset substrate.

On more complex structures than $50 \Omega$ lines such as Wilkinson power dividers, RF performances of both type of multilayer assemblies keep very similar as illustrated on Fig. 7. In each case, return losses and isolation between outputs are very good (respectively lower than $20 \mathrm{~dB}$ and $-25 \mathrm{~dB}$ nearly on the whole band). Here, insertion losses are slightly higher for the thermosetbased board probably because the Wilkinson test structure has longer copper traces than the $50 \Omega$ line and as a consequence is more sensitive to the higher dielectric losses from thermoset substrates.

In summary, the thermoset based multilayer board and the PTFE based one exhibit rather similar RF performances in spite of the slightly lower dielectric properties from thermoset laminates. Such electrical results confirm the expected better accuracy allowed by thermoset laminates and prepreg during multilayer board manufacturing, resulting in more homogeneous and more thermo-mechanically stable structures than with their PTFE-based counterparts. Such benefits should be even more sensitive for more complex multilayer circuits than the ones evaluated here, i.e. structures with up to 10 layers or with mixed dielectric architecture.
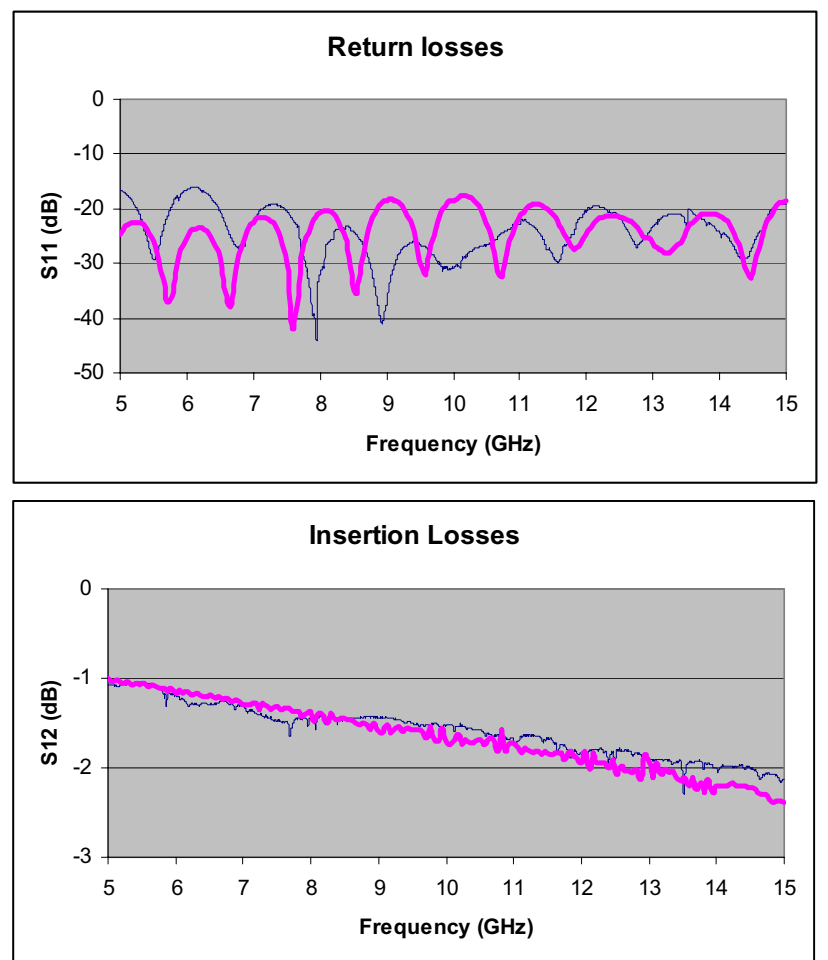

Fig. 6. S-parameters measurement of a $50 \Omega$ lines in stripline configuration on a 3 layers PTFE-based (thin line) and a 6 layers thermoset-based (thick line) RF PCB. 

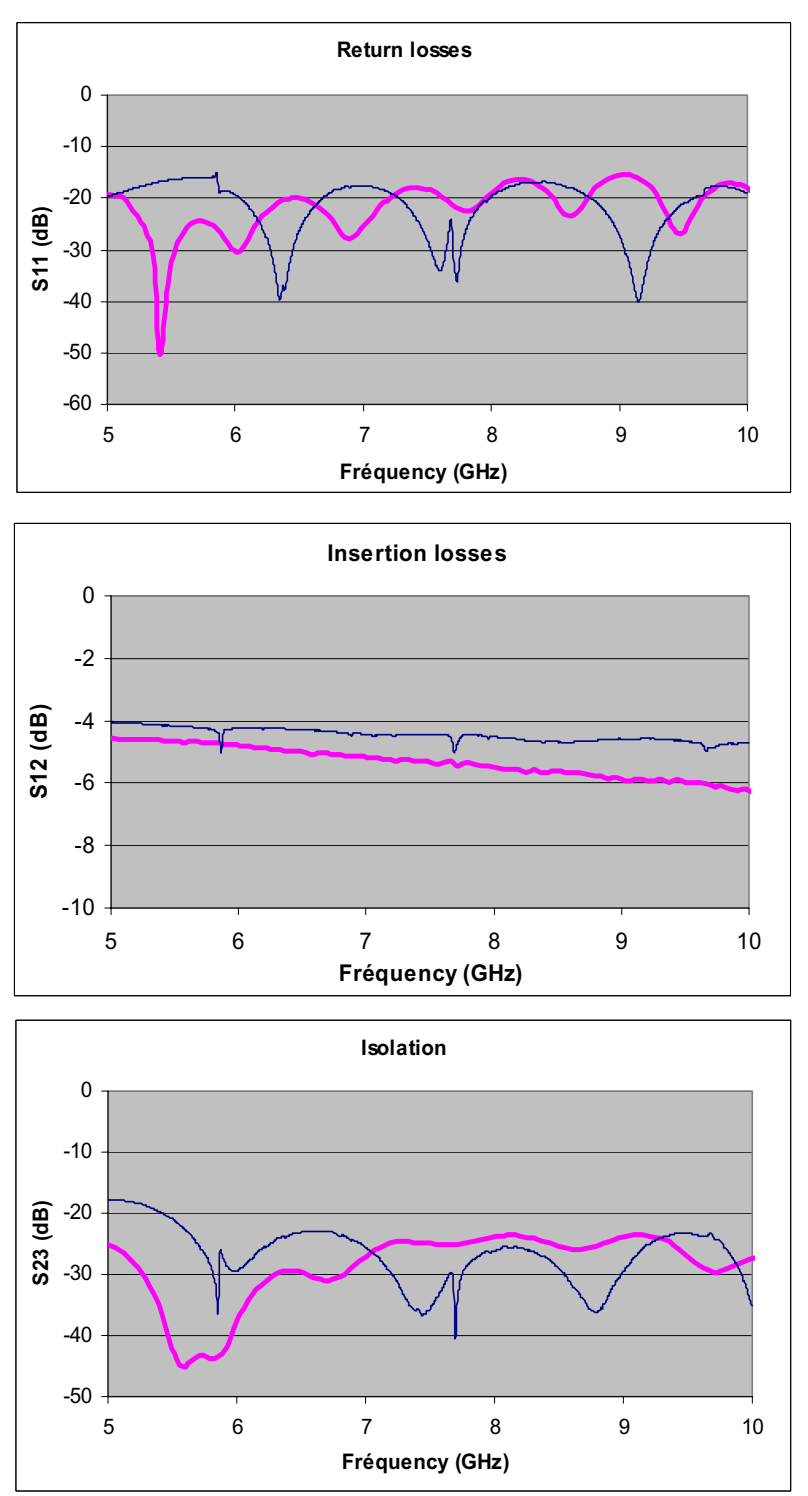

Fig. 7.S-parameters measurements of a Wilkinson power divider on a 3 layers PTFE-based (thin line) and a 6 layers thermoset-based (thick line) RF PCB.

\section{CONCLUSION}

Complex multilayer RF Printed Circuit Boards with embedded resistors have been designed, manufactured and tested in order to benchmark PTFE and thermoset based substrates and prepregs. Advantages and drawbacks of both technologies are discussed and thermoset laminates are demonstrated to offer similar RF performances to their PTFE-based counterparts but with easier and more accurate manufacturing processes. In parallel, an original and very reproducible interconnection technique based on "RF openings" machined in the edges of the circuit has also been demonstrated. This method offer new solutions for interconnections of multilayer boards to their environment without any degradations of the RF signal. By combining all these technological solutions, very good RF performances have been demonstrated on different test structures up to $15 \mathrm{GHz}$.

\section{ACKNOWLEDGEMENT}

The authors would like to acknowledge the French Space Agency (CNES) for funding part of this work.

\section{REFERENCES}

[1] J.L. Cazaux, J.C. Cayrou, C. Miquel, C. Debarge, S. Geogre, R. Barbaste, F. Bodereau, P. Chabbert, J. Maynard, "New Generation of Ka-Band Equipment for Telecommunication Satellites" in Proceedings of the 34st European Microwave Conference, Amsterdam, The Netherlands, 2004, pp. 325-328.

[2] C. Drevon, G. Naudy, D. Roques, L. Albo, J.L. Lacoste, B. Cogo, F. Dubos, A. Coello Vera, "Multilayer Printed Circuit Board at 12-14 GHZ with MCMs and MMICS" in Proceedings of 1999 Wireless Circuits, Interconnection and Assembly Workshop, Oct. 24-2, 1999 - Gold Canyon. 